\title{
On the eccentric adjacency index of graphs
}

\author{
Yasar Nacaroglu
}

Department of Mathematics, Faculty of Science and Arts, Kahramanmaras Sutcu Imam University, Kahramanmaras, Turkey

Received: 7 June 2018, Accepted: 29 July 2018

Published online: 8 August 2018.

Abstract: In this paper, we present some bounds for the eccentric adjacency index. Also we will calculate the eccentric adjacency index of some thorny graphs.

Keywords: Eccentric-adjacency index, Zagreb indices, Thorny graphs.

\section{Introduction}

Let $G$ be a $n$ vertices graph with vertex set $V(G)$ and edge set $E(G)$. The degree of a vertex $u$ in a graph $G$, denoted by $d(u)$, is the number of proper edges incident on $u$. A graph in which every vertex has degree $k$ is $k$ regular.

The distance between two vertices in a graph is the length of the shortest walk between them. The eccentricity of a vertex $u$ in a graph $G$, denoted by $e_{G}(u)$, is the maximum distance from u to any vertex. The radius of a connected graph is the minimum eccentricity. The diameter of a connected graph is the maximum eccentricity. Diameter and radius of a graph is denoted by $D=D(G)$ and $r=r(G)$, respectively. The total eccentricity [1] of a graph is denoted by $\zeta(G)$ and is equal to sum of eccentricities of all the vertices of the graph. The total eccentricity is

$$
\zeta(G)=\sum_{u \in V(G)} e_{G}(u)
$$

where $e_{G}(u)$ is the eccentricity of the vertex $u$. A central vertex in a graph is a vertex whose eccentricity equals the radius of the graph. A graph in which every vertex has same eccentricity is self centered graph. A pendant vertex is a vertex of degree one.

As usual, star graph, complete graph, compete bipartite graph and cycle on $n$ vertices is denoted $S_{n}, K_{n}, K_{m, n}$ and $C_{n}$, respectively. The minimum vertex degree and the maximum vertex degree in a graph is denoted by $\Delta$ and $\delta$, respectively.For other undefined notations and terminology from graph theory, the readers are referred to [7].

Gutman and Trinajstic[9,10] introduced Zagreb indices. The first and second Zagreb indices of G are denoted by $M_{1}(G)$ and $M_{2}(G)$, respectively and defined as:

$$
M_{1}(G)=\sum_{u \in V(G)} d_{G}^{2}(u) \quad \text { and } \quad M_{2}(G)=\sum_{u v \in E(G)} d_{G}(u) d_{G}(v)
$$


The eccentric connectivity index of a graph $G$ is defined as [19]

$$
\xi^{c}(G)=\sum_{u \in V(G)} d(u) e(u)
$$

For more results of eccentric connectivity index, we refer our reader to [16,19,22].

In 2001, Gupta et al.[8] proposed a topological descriptor that adjacency-cut distance based. Eccentric adjacency index (also known as Ediz eccentric connectivity index see [4]) is denoted as $\xi^{a d}(G)$ and is defined as :

$$
\xi^{a d}(G)=\sum_{u \in V(G)} \frac{S(u)}{e(u)}
$$

where $S_{u}$ is sum degrees of vertices adjacent to vertex $u$. Gupta et al.[8] investigated the power of eccentric adjacent index in the estimination of anti-HIV activity. The accuracy of prediction $\xi^{a d}(G)$ is more than ninety percent. Thus $\xi^{\text {ad }}(G)$ proposes a vast potential for QSAR/QSPR studies.

Ediz [4] investigated some mathematical properties of Ediz eccentric conenctivity index. Sharafdini et al.[18] investigated the eccentric adjacency index of several infinite class of fullerenes. Wu et al.[21] studied the Ediz eccentric connectivity index of some molecular structures. Farahani [5,6] determined Ediz eccentric connectivity index of molecular graph circumcoronene series of benzenoid $H_{k}$. Malik [14] computed eccentric adjacent index of the join and corona products of graphs.

In this paper we propose to investigate some mathematical properties of this novel connectivity index. In this paper, we will give some bounds for the eccentric-adjacency index. Also we will present eccentric-adjacency index of thorn graphs obtained some well-defined graphs.

\section{Main results}

In this section, we give some bounds for the eccentric-adjacency index. After that we will obtain eccentric-adjacency indices of thorny star graphs, complete graphs, bipartite complete graphs and cycles.

We first give the following lemma which will be used in this paper.

Lemma 1. Let $G$ be a graph. Then

$$
\sum_{u \in V(G)} S(u)=M_{1}(G)
$$

Proof. When calculating this sum, we can see that each of the degree of $u \in V(G)$ vertex is calculated in this sum up to degree of its. So we get the following the result.

$$
\sum_{u \in V(G)} S(u)=\sum_{u \in V(G)} d(u) \cdot d(u)=\sum_{u \in V(G)} d^{2}(u)=M_{1}(G) .
$$

Theorem 1. We have for any graph

$$
\frac{M_{1}(G)}{D} \leq \xi^{a d}(G) \leq \frac{M_{1}(G)}{r} .
$$

Furthermore, the equality in each inequality is attained if and only if $G$ is self centered graph. 
Proof. Since $G$ be a simple connected graph of order $n$, diameter $D$ and radius $r$, then By Lemma 1 and the fact $r \leq e(u) \leq$ $D$, we have

$$
\xi^{a d}(G)=\sum_{u \in V(G)} \frac{S(u)}{e(u)} \leq \sum_{u \in V(G)} \frac{S(u)}{r}=\frac{1}{r} \sum_{u \in V(G)} S(u)=\frac{M_{1}(G)}{r} .
$$

Now suppose that equality holds in (1). Thus we have $e(u)=r$ for any $u \in V(G)$. Hence the equality holds if and only if $G$ is self centered graph. Similarly, we get

$$
\xi^{a d}(G) \geq \frac{M_{1}(G)}{D}
$$

The equality holds if and only if $G$ is self centered graph.

Lemma 2. [12] Let $G$ be a graph with $n$ vertices and $m$ edges. Then

$$
M_{1}(\bar{G})=M_{1}(G)+n(n-1)^{2}-4 m(n-1) .
$$

Corollary 1. Let $G$ be a connected graph with $n \geq 4$ vertices for which the complement $\bar{G}$ is also connected. Then

$$
\xi^{a d}(G)+\xi^{a d}(\bar{G}) \leq M_{1}(G)+\frac{n(n-1)^{2}}{2}-2 m(n-1),
$$

with equality holds if and only if $G$ and $\bar{G}$ are self centered graphs with radius two.

Proof. Let $M_{1}(G)$ and $M_{1}(\bar{G})$ be the first Zagreb indices of $G$ and $\bar{G}$,respectively. Since both $G$ and $\bar{G}$ are connected, each has radius at least two, and then by Lemma 2 and Theorem 1 ,

$$
\xi^{a d}(G)+\xi^{a d}(\bar{G}) \leq \frac{M_{1}(G)+M_{1}(\bar{G})}{2}=M_{1}(G)+\frac{n(n-1)^{2}}{2}-2 m(n-1),
$$

with equality holds if and only both if $G$ and $\bar{G}$ are self centered, and $r(G)=r(\bar{G})=2$.

Remark. By some basic elementary calculations, one may see that the bounds (1) are better than the results [4, Theo. 1 and Theo. 2].

Lemma 3. Radon Inequality)[17]. For every real numbers $p>0, x_{k} \geq 0, a_{k}>0$, for $1 \leq k \leq n$, the following inequality holds true:

$$
\sum_{k=1}^{n} \frac{x_{k}^{p+1}}{a_{k}^{p}} \geq \frac{\left(\sum_{k=1}^{n} x_{k}\right)^{p+1}}{\left(\sum_{k=1}^{n} a_{k}\right)^{p}}
$$

The equality holds if and only if $\frac{x_{1}}{a_{1}}=\frac{x_{2}}{a_{2}}=\cdots=\frac{x_{n}}{a_{n}}$.

Theorem 2. We have for any graph $G$

$$
\xi^{a d}(G) \geq \frac{M_{1}(G)+\delta^{2} n(n-1)}{\zeta(G)}
$$

and the equality is attained if and only if $G$ is a regular self centered graph.

Proof. Using (2) we get

$$
\xi^{a d}(G)=\sum_{u \in V(G)} \frac{(\sqrt{S(u)})^{2}}{e(u)} \geq \frac{\left(\sum_{u \in V(G)} \sqrt{S(u)}\right)^{2}}{\sum_{u \in V(G)} e(u)}=\frac{M_{1}(G)+2 \sum_{1 \leq i<j \leq n} \sqrt{S(i) S(j)}}{\zeta(G)}
$$


Since any for $u \in V(G) S(u) \geq \delta^{2}$, we have

$$
\xi^{a d}(G) \geq \frac{M_{1}(G)+\delta^{2} n(n-1)}{\zeta(G)}
$$

By Lemma 2, In (3) equality holds if and only if $G$ is a regular self centered graph.

Remark. By some basic elementary calculations, one may see that the bounds (3) are better than the results [4, Theo. 2]. In theorem 2, by applying $e(u) \leq n-d(u)$ [22] for all of $u \in V(G)$ we get the following result.

Corollary 2. et $G$ be a simple connected graph with $n$ vertices and $\delta$ minimum degrees. Then

$$
\xi^{a d}(G) \geq \frac{M_{1}(G)+\delta^{2} n(n-1)}{n^{2}-2 m},
$$

with equality holds if and only if $G$ is a regular self centered graph.

Lemma 4. (Chebyshev's inequality)[13] Let $a_{1} \geq a_{2} \geq \cdots a_{n}$ and $b_{1} \geq b_{2} \geq \cdots b_{n}$ be real numbers. Then

$$
n \sum_{i=1}^{n} a_{i} b_{i} \geq\left(\sum_{i=1}^{n} a_{i}\right)\left(\sum_{i=1}^{n} b_{i}\right)
$$

with equality holding if and only if $a_{1}=a_{2}=\cdots=a_{n}$ and $b_{1}=b_{2}=\cdots=b_{n}$.

Theorem 3. For any connected graph $G$ on $n$ vertices

$$
\xi^{a d}(G) \geq \frac{n M_{1}(G)}{\zeta(G)}
$$

The equality holds if and only if $G$ regular self centered graph.

Proof. By Lemma 4, for $i=1,2, \cdots, n$ by taking $a_{i}=S(i)$ and $b_{i}=e(i)$ we get

$$
n \sum_{i=1}^{n} \frac{S(i)}{e(i)} \geq\left(\sum_{i=1}^{n} S(i)\right)\left(\sum_{i=1}^{n} \frac{1}{e(i)}\right) .
$$

Also by aritmetic-harmonic mean inequality, we have

$$
\sum_{i=1}^{n} \frac{1}{e(i)} \geq \frac{n^{2}}{\sum_{i=1}^{n} e(i)}=\frac{n^{2}}{\zeta(G)}
$$

As a result by (5) and (6), we have

$$
\xi^{a d}(G) \geq \frac{n M_{1}(G)}{\zeta(G)}
$$

with equality holds if and only if $G$ regular self centered graph.

Corollary 3. Let $G$ be a connected graph of order $n$. We have $e(u) \leq n-d_{u v}$ for $u \in V(G)$. Then by Theorem 3 we get

$$
\xi^{a d}(G) \geq \frac{n M_{1}(G)}{n^{2}-2 m} .
$$


Remark. By some basic elementary calculations, one may see that the bounds in (4) are better than the results [4, Theo. 3]. Also, if $G$ regular self centered graph, (4) and [4, Theorem 3] bounds are the same.

Proposition 1. Let $W_{n}$ and $B_{n}$ denote the graphs of the pyramid and the bipyramid with $n$-gonal base, $n \geq 3$. Then

$$
\xi^{a d}\left(W_{n}\right)=\frac{n^{2}+12 n}{2} \quad \text { and } \quad \xi^{a d}\left(B_{n}\right)=n^{2}+8 n .
$$

Let $K_{n}-k e$ be the graph obtained from $K_{n}$ by deleting $k$ independent edges for $0 \leq k \leq\left\lfloor\frac{n}{2}\right\rfloor$.

Theorem 4. Let $G$ be a connected graph.. Let $k$ be the number of vertices with eccentricity 1 in graph $G$. Then

$$
\xi^{a d}(G) \leq \frac{M_{1}(G)+k(2 m-n+1)}{2},
$$

with equality if and only if $G \cong K_{k} \vee\left(K_{n-k}-\frac{n-k}{2} e\right)$, where $n-k$ is even.

Proof. $T=\left\{v_{0}, v_{1}, \ldots, v_{k}\right\}$ be the set of vertices with eccentricity 1 . Then $e(u) \geq 2, d(u) \leq n-2$ for any $u \in V(G) \backslash T$. By the definition eccentric-adjacency index, we have

$$
\xi^{a d}(G)=\sum_{v_{i} \in T} \frac{S\left(v_{i}\right)}{e(i)}+\sum_{u \in V(G) \backslash T} \frac{S(u)}{e(u)} \leq[2 m-(n-1)] k+\frac{\sum_{u \in V(G) \backslash T} S(u)}{2} .
$$

Since $\sum_{u \in V(G) \backslash T} S(u)+\sum_{v_{i} \in T} S\left(v_{i}\right)=\sum_{u \in V(G)} S(u)$, we have $\sum_{u \in V(G) \backslash T} S(u)=M_{1}(G)-\sum_{v_{i} \in T} S\left(v_{i}\right)$. So we get

$$
\xi^{a d}(G) \leq \frac{M_{1}(G)+k(2 m-n+1)}{2} .
$$

The above equality holds if and only if $G \cong K_{k} \vee\left(K_{n-k}-\frac{n-k}{2} e\right)$, where $n-k$ is even.

Theorem 5. Let $G$ be a simple connected graph on $n$ vertices with $m$ edges. Let

$$
a=\left\lfloor\frac{2 n-1-\sqrt{(2 n-1)^{2}-8 m}}{2}\right\rfloor
$$

be the largest integer satisfying that $x^{2}-(2 n-1) x+2 m \geq 0$. Then

$$
\xi^{a d}(G) \leq \frac{M_{1}(G)+a(2 m-n+1)}{2},
$$

with equality if and only if $G \cong K_{a} \vee\left(K_{n-a}-\frac{n-a}{2} e\right)$, where $n-a$ is even.

Proof. Let be $k$ is the number of vertices with eccentricity 1 in graph G. Then degree of these vertices be $n-1$. Since $2 m=\sum_{u \in V(G)} \geq k(n-1)+k(n-k)$, so implies that $k \leq a$. Therefore by Theorem 4 , we get

$$
\xi^{a d}(G) \leq \frac{M_{1}(G)+k(2 m-n+1)}{2} \leq \frac{M_{1}(G)+a(2 m-n+1)}{2},
$$

with equality if and only if $G \cong K_{a} \vee\left(K_{n-a}-\frac{n-a}{2} e\right)$, where $n-a$ is even. 
Theorem 6. Let $G$ be a simple connected graph. Then

$$
E(G) \leq M_{1}(G)
$$

The equality holds if and only if $G$ is complete graph with $n$ vertices.

Proof. We have

$$
\xi^{a d}(G)=\sum_{u \in V(G)} \frac{S(u)}{e(u)} \leq \sum_{u \in V(G)} S(u)=M_{1}(G)
$$

Suppose that equality holds in (7). Then we have $e(v)=r$ for any $v \in V(G)$. Hence we get $G \cong K_{n}$. Conversely, if $G \cong K_{n}$, we have $E\left(K_{n}\right)=M_{1}\left(K_{n}\right)=n(n-1)^{2}$. Proof is completed.

Theorem 7. Let $G$ be a simple connected graph. Then

$$
\xi^{a d}(G) \geq \frac{k n^{2}}{\zeta(G)}
$$

where $k=\min (S(u), u \in V(G))$.

Proof. We have

$$
\xi^{a d}(G)=\sum_{u \in V(G)} \frac{S(u)}{e(u)} \geq \sum_{u \in V(G)} \frac{k}{e(u)}
$$

Also by aritmetic-harmonic mean inequality, we get

$$
\sum_{u \in V(G)} \frac{1}{e(u)} \geq \frac{n^{2}}{\sum_{u \in V(G)} e(u)}=\frac{n^{2}}{\zeta(G)}
$$

By (8) and (9), we have

$$
\xi^{a d}(G) \geq \frac{k n^{2}}{\zeta(G)}
$$

Definition 1. [11]. Let $p_{1}, p_{2}, \cdots, p_{n}$ be non-negative integers. The thorn graph of the graph $G$, with parameters $p_{1}, p_{2}, \cdots, p_{n}$, is obtained by attaching $p_{i}$ new vertices of degree one to the vertex $u_{i}$ of the graph $G, i=1,2, \cdots, n$. The thorn graph of the graph $G$ will be denoted by $G^{*}$.

Recently, various studies on thorn graphs have done by some researchers $[2,3,15,20]$. Now, we will examine the eccentric adjacency indices of some specific thorn graphs.

Theorem 8. Let $S_{n}$ and $S_{n}^{*}$ denote star graph and its thorn graph with $n$ vertices, respectively. Then

$$
\xi^{a d}\left(S_{n}^{*}\right)=\frac{13}{12} A_{1}+\frac{1}{4} A_{2}+\frac{(n-1)(2 n+1)}{6}+\frac{8 n-15}{4} p_{1}+\frac{1}{12} p_{1}^{2},
$$

where $p_{1}$ is the number of pendant vertices added to the central vertex of $S_{n}$. Also $A_{1}=\sum_{i=1}^{n} p_{i}, A_{2}=\sum_{i=1}^{n} p_{i}^{2}$.

Proof. Let $S_{n}^{*}$ be the thorny star graph. Then we have $d\left(u_{1}\right)=n-1+p_{1}, d\left(u_{i}\right)=1+p_{i}, i=2,3, \ldots, n, e\left(u_{1}\right)=2, e\left(u_{i}\right)=3$, $i=2,3, \ldots, n, d\left(u_{i j}\right)=1, e\left(u_{i j}\right)=4$, for $i=2,3, \ldots, n, j=2,3, \ldots, p_{i}, e\left(u_{1 j}\right)=3$, for $j=1,2, \ldots, p_{1}$. So, we get the following 
result

$$
\begin{aligned}
\xi^{a d}\left(S_{n}^{*}\right) & =\frac{S\left(u_{1}\right)}{e\left(u_{1}\right)}+\sum_{i=2}^{n} \frac{S\left(u_{i}\right)}{e\left(u_{i}\right)}+\sum_{j=1}^{p_{1}} \frac{S\left(u_{1 j}\right)}{e\left(u_{1 j}\right)}+\sum_{i=2}^{n} \sum_{j=1}^{p_{1}} \frac{S\left(u_{i j}\right)}{e\left(u_{i j}\right)} \\
& =\frac{p_{1}}{2}+\sum_{i=2}^{n} \frac{p_{i}+1}{2}+\sum_{i=2}^{n} \frac{p_{1}+p_{i}+n-1}{3}+\sum_{j=1}^{p_{1}} \frac{p_{1}+n-1}{3}+\sum_{i=2}^{n} \sum_{j=1}^{p_{i}} \frac{\left(p_{i}+1\right) p_{i}}{4} \\
& =\frac{13}{12} A_{1}+\frac{1}{4} A_{2}+\frac{(n-1)(2 n+1)}{6}+\frac{8 n-15}{4} p_{1}+\frac{1}{12} p_{1}^{2}
\end{aligned}
$$

Theorem 9. The eccentric-adjacency index of thorny cycle $C_{n}^{*}$ is given by

$$
\xi^{a d}\left(C_{n}^{*}\right)=\frac{2 A_{1}+4 n}{\left\lfloor\frac{n}{2}\right\rfloor+1}+\frac{2 A_{1}+A_{2}}{\left\lfloor\frac{n}{2}\right\rfloor+2}
$$

where $A_{1}=\sum_{i=1}^{n} p_{i}$ and $A_{2}=\sum_{i=1}^{n} p_{i}^{2}$

Proof. Let $C_{n}^{*}$ be the thorny cycle graph. Then we have $d\left(u_{i}\right)=p_{i}+2, d\left(u_{i j}\right)=1, e\left(u_{i}\right)=\left\lfloor\frac{n}{2}\right\rfloor+1$ and $e\left(u_{i j}\right)=\left\lfloor\frac{n}{2}\right\rfloor+2$ for $i=1,2, \ldots, n ; j=1,2, \ldots, p_{i}$. Thus we get

$$
\xi^{a d}\left(C_{n}^{*}\right)=\sum_{i=1}^{n} \frac{S\left(u_{i}\right)}{e\left(u_{i}\right)}+\sum_{i=1}^{n} \sum_{j=1}^{p_{i}} \frac{S\left(u_{i j}\right)}{e\left(u_{i j}\right)}=\sum_{i=1}^{n} \frac{2 p_{i}+4}{\left\lfloor\frac{n}{2}\right\rfloor+1}+\sum_{i=1}^{n} \sum_{j=1}^{p_{i}} \frac{\left(p_{i}+2\right) p_{i}}{\left\lfloor\frac{n}{2}\right\rfloor+2}=\frac{2 A_{1}+4 n}{\left\lfloor\frac{n}{2}\right\rfloor+1}+\frac{2 A_{1}+A_{2}}{\left\lfloor\frac{n}{2}\right\rfloor+2} .
$$

Theorem 10. The eccentric-adjacency index of thorny complete graph $K_{n}^{*}$ is given by

$$
\xi^{a d}\left(K_{n}^{*}\right)=\frac{5 n-2}{3} A_{1}+\frac{1}{3} A_{2}+\frac{n(n-1)^{2}}{2}
$$

where $A_{1}=\sum_{i=1}^{n} p_{i}, A_{2}=\sum_{i=1}^{n} p_{i}^{2}$.

Proof. Let $K_{n}^{*}$ be the thorny complete graph. Let the vertices of $K_{n}$ are denoted by $u_{i}$. Also, let the vetices $u_{i}$ in $K_{n}$ are denoted by $u_{i j}, i=1,2, \ldots, n ; j=1,2, \ldots, p_{i}$. Therefore we have $d\left(u_{i}\right)=p_{i}+n-1, d\left(u_{i j}\right)=1, e\left(u_{i}\right)=2, e\left(u_{i j}\right)=3$ for $i=1,2, \ldots, n ; j=1,2, \ldots, p_{i}$. Thus the eccentric-adjacency index of $K_{n}^{*}$ is given by

$$
\xi^{a d}\left(K_{n}^{*}\right)=\sum_{i=1}^{n} \frac{S\left(u_{i}\right)}{e\left(u_{i}\right)}+\sum_{i=1}^{n} \sum_{j=1}^{p_{i}} \frac{S\left(u_{i j}\right)}{e\left(u_{i j}\right)}=\sum_{i=1}^{n} \frac{A_{1}+(n-1)^{2}}{2}+\sum_{i=1}^{n} \sum_{j=1}^{p_{i}} \frac{p_{i}+n-1}{3}=\frac{5 n-2}{3} A_{1}+\frac{1}{3} A_{2}+\frac{n(n-1)^{2}}{2} .
$$

Let $K_{m, n}$ be a complete bipartite graph with vertex set $\left\{u_{1}, u_{2}, \ldots, u_{m}, v_{1} v_{2}, \ldots, v_{n}\right\}$. Let $K_{m, n}^{*}$ be thorny complete bipartite graph obtained from $K_{m, n}$ by attaching pendant vertices $p_{i}$ and $p_{k}^{*}$ to $u_{i}$ and $v_{k}$. Let the newly attached pendant vertices are denoted by $u_{i j}$ and $v_{k l}$, where $i=1,2, \ldots, m ; j=1,2, \ldots, p_{i} ; k=1,2, \ldots, n ; l=1,2, \ldots, p_{i}^{*}$.

Theorem 11. The eccentric-adjacency index of thorny cycle $K_{m, n}^{*}$ is given by

$$
\xi^{a d}\left(K_{m, n}^{*}\right)=\frac{7 n+4}{12} A_{1}+\frac{7 m+4}{12} A_{1}^{*}+\frac{A_{2}+A_{2}^{*}}{4}+\frac{n m(n+m)}{3},
$$

where $A_{1}=\sum_{i=1}^{m} p_{i}, A_{1}^{*}=\sum_{i=1}^{n} p_{i}^{*}, A_{2}=\sum_{i=1}^{m} p_{i}^{2}$ and $A_{2}^{*}=\sum_{i=1}^{n}\left(p_{i}^{*}\right)^{2}$. 
Proof. The degree and eccentricity of the vertices of $K_{m, n}^{*}$ are given by $d\left(u_{i}\right)=p_{i}+n, d\left(v_{k}\right)=p_{k}^{*}+m, d\left(u_{i j}\right)=d\left(v_{k l}\right)=1$, $e\left(u_{i}\right)=3, e\left(v_{k}\right)=3, e\left(u_{i j}\right)=4, e\left(v_{k l}\right)=4$ for $i=1,2, \ldots, m ; j=1,2, \ldots, p_{i} ; k=1,2, \ldots, n ; l=1,2, \ldots, p_{i}^{*}$. Then we have

$$
\begin{aligned}
\xi^{a d}\left(K_{m, n}^{*}\right) & =\sum_{i=1}^{m} \frac{p_{i}+A_{1}^{*}+n m}{3}+\sum_{i=1}^{n} \frac{p_{i}^{*}+A_{1}+n m}{3}+\sum_{i=1}^{m} \sum_{j=1}^{p_{i}} \frac{p_{i}+n}{4}+\sum_{i=1}^{m} \sum_{j=1}^{p_{i}^{*}} \frac{p_{i}^{*}+m}{4} \\
& =\frac{A_{1}+m A_{1}^{*}+n m^{2}}{3}+\frac{A_{1}^{*}+n A_{1}+n^{2} m}{3}+\frac{A_{2}+n A_{1}}{4}+\frac{A_{2}^{*}+m A_{1}^{*}}{4} \\
& =\frac{7 n+4}{12} A_{1}+\frac{7 m+4}{12} A_{1}^{*}+\frac{A_{2}+A_{2}^{*}}{4}+\frac{n m(n+m)}{3} .
\end{aligned}
$$

\section{Competing interests}

The authors declare that they have no competing interests.

\section{Authors' contributions}

All authors have contributed to all parts of the article. All authors read and approved the final manuscript.

\section{References}

[1] P. Dankelmann, W. Goddard and C. S. Swart, The Average Eccentricity of a Graph and Its Subgraphs, Util. Math. 65 (2004) $41-51$.

[2] N. De, Aughmented eccentric connectivity index of some thorn graph, Int. J. Appl. Math. Res. 1(4) (2012) 671-680.

[3] N. De, Sk.Md.A. Nayeem and A. Pal, Connective eccentricity index of some thorny graphs, Ann. Pure Appl. Math. 7(1) (2014) 59-64.

[4] S. Ediz, On the Ediz eccentric connectivity index of a graph, Optoelectron Adv. Mat.-Rapid Commun. 5(11) (2011) $1263-124$.

[5] M.R. Farahani, The Ediz eccentric connectivity index and the total eccentric connectivity index benzonoid system, J. Chem. Acta 2 (2013) 22-25.

[6] M.R. Farahani, M.K. Jamil and M.R.R. Kanna, About the Ediz eccentric connectivity index of linear polycene parallelogram benzenoid, Int. J. Sci. Engrg. Res. 7(1)) (2016) 1469-1475.

[7] J.L. Gross and J. Yellen, Handbook of graph theory, Chapman Hall, CRC Press (2004).

[8] S. Gupta, M. Singh and A.K. Madan, Predicting anti-HIV activity: computational approach using a novel topological descriptor, J. Comput. Aided. Mol. Des. 15 (7) (2001) 671-678.

[9] I. Gutman and N. Trinajstić, Graph theory and molecular orbitals, Total $\pi$ electron energy of alternant hydrocarbons, Chem. Phys. Lett. 17 (1972) 535-538.

[10] I. Gutman, B. Ruščić, N. Trinajstić, C.F. Wilcox, Graph theory and molecular orbitals. XII. Acyclic polyenes., J Chem Phys. 62 (1975) 3399-3405.

[11] I Gutman, Distance of thorny graphs, Publ. I. Math. 77 (1998) 31-36.

[12] I. Gutman, B. Furtula, Ž K. Vukićević and G. Popivoda, On Zagreb Indices and Coindices, MATCH Commun Math Comput Chem. 74 (2015) 5-16.

[13] G.H. Hardy, J.E. Littlewood and G. Pólya, Inequalities. second edition, Cambridge Mathematical Library. Cambridge: Cambridge University Press (2001)

[14] M.A. Malik, Two degree-distance based topological descriptors of some product graphs, Discrete Appl. Math. 236 (2018) 315 -328.

[15] U. Mary, A.K. Therese, M.J. Seles, R. Jayasree and J. Kok, On Zagreb indices and eccentric connectivity index of certain thorn graphs, Internat. J. Pure Appl. Math. 106 (3) (2016) 909-921.

[16] Y. Nacaroglu and A.D. Maden, On the eccentricic connectivity index of unicyclic graph, Iranian J. Math. Chem. 9 (1) (2018) 47-56. 
[17] J. Radon, Uber die absolut additiven Mengenfunktionen, Wiener Sitzungsber 122 (1913) 1295-1438.

[18] R. Sharafdini and M. Safazadeh, On eccentric adjacency index of several infinite classes of fullerenes, British J. Math. Comput. Sci. 12 (5) (2016) 1-11.

[19] V. Sharma, R. Goswami and A.K. Madan, Eccentric connectivity index; Anovel highly discriminating topological descriptor for structure-property and structure-activity studies, J. Chem. Inf. Compt. Sci. 37 (2) (1997) 273-282.

[20] Y.B. Venkatakrisnan, S. Balanchandran and K. Kannan, On the eccentric connectivity index of generalized thorn graphs, Natl. Acad. Sci. Lett. 38 (2) (2015) 165-168.

[21] H. Wu, M.K. Siddiqui, B. Zhao, J. Gan and W. Gao, Computing the Ediz eccentric connectivity index of discrete dynamic structures, Open Phys. 15 (2017) 354-359.

[22] B. Zhou and Z. Du, On eccentric connectivity index, Match. Commun. Math. Comput. Chem. 63 (2010) 181-198. 